Journal of

Women's Health and Gynecology

\title{
Adherence to the Single-Dose Methotrexate Protocol for the Management of Ectopic Pregnancy in an Inner City Population: A retrospective cohort and protocol analysis
}

Rachel K. Scott ${ }^{1, *}$, Charelle M. Carter-Brooks², Graysan Kirk Southern ${ }^{3}$, Sameer Desale ${ }^{4}$, E. Britton Chahine $^{5}$

${ }^{1}$ MedStar Health Research Institute, 100 Irving Street NW, East Building, 108 Washington, DC

${ }^{2}$ Department of Obstetrics, Gynecology and Reproductive Sciences Magee, Women's Hospital of the University of

Pittsburgh Medical Center

${ }^{3}$ Department of Obstetrics \& Gynecology, George Washington University

${ }^{4}$ MedStar Health Research Institute, 100 Irving Street NW, East Building, 108 Washington, DC

${ }^{5}$ Department of Gynecology \& Obstetrics, Emory University

${ }^{*}$ Corresponding author: Rachel K. Scott, MedStar Health Research Institute, 100 Irving Street NW, East Building, 108 Washington, DC 20010; Tel: 617-513-9930; E-mail: rachelkscott@gmail.com or rachel.k.scott@medstar.net

Received Date: November 01, 2016; Accepted Date: November 25, 2016; Published Date: November 28, 2016

Citation: Rachel K. Scott, et al. (2016) Adherence to the Single-Dose Methotrexate Protocol for the Management of Ectopic Pregnancy in an Inner City Population: A retrospective cohort and protocol analysis. J Womens Health Gyn 1: 1-10.

\section{Abstract}

Objective: To evaluate protocol adherence, treatment success, and associated morbidity to single dose methotrexate (MTX) ectopic pregnancy treatment in an urban population.

Methods: We performed a retrospective chart review of patients treated for ectopic pregnancy with MTX from January 2009 to December 2012.

Results: A total of 117 patients met inclusion criteria. Follow up trends were nonspecific. Ninety-three percent of patients followed up for at least one additional blood draw: day 4 (39.3\%), day 5 (32.5\%), day 7 (25.6\%) and day 8 (34.2\%). Standard MTX follow-up was $16.2 \%$. No significant differences in morbidity or treatment failure were associated with patterns of follow-up.

Conclusions: Adherence to standard MTX protocol in our urban population was low. Neither success of treatment nor morbidity was adversely affected by non-adherence to current standard protocol.

Keywords: Methotrexate; Ectopic pregnancy; Adherence

Capsule: Adherence to the standard single-dose methotrexate protocol for treatment of ectopic pregnancy is poor; alternate protocols appear safe and may facilitate improved adherence.

(C)2016 The Authors. Published by the JScholar under the terms of the Creative Commons Attribution License http://creativecommons.org/licenses/ by/3.0/, which permits unrestricted use, provided the original author and source are credited. 


\section{Introduction}

Methotrexate (MTX) is a common medical treatment of unruptured ectopic pregnancy (EP). The standard singledose treatment regimen administers a weight -based MTX injection on day 1 and follows human chorionic gonadotropin (hCG) levels on days 1, 4 and 7. Successful treatment is defined as a $\geq 15 \%$ decrease between day 1 and day 4 or day 7 [1]. Adherence to this protocol in inner city populations is poor; less than $20 \%$ of patients adhere to the follow-up protocol and greater than $50 \%$ are lost to follow-up [2]. Poor adherence to follow-up after MTX raises concerns regarding the utility and safety of the current standard of care protocol. Several studies have validated alternative protocols which define treatment success as a decrease in hCG of $\geq 20 \%$ between days 1 and 4 , or $\geq 50 \%$ days 1 and 7 [3].

Our overarching hypothesis was that, as adherence to the standard MTX protocol was likely poor, alternate protocols with fewer follow-up visits may facilitate and improve patient adherence. Our primary objective was to assess patient adherence to the standard MTX protocol follow-up and to assess if adherence would have been improved with alternate protocols in our inner city population. Our secondary objective was to evaluate for morbidity associated with non-adherence to the standard protocol. We hypothesized that the majority of our patients were not adherent to the standard protocol and more likely to only follow up only once. We further hypothesized there would be no difference in outcomes between patients who followed up on day 4 or on day 7 compared to the standard protocol of both day 4 and 7 .

\section{Material and Methods}

MedStar Washington Hospital Center (MWHC) is a not-for-profit, 926-bed, major teaching and research hospital in inner city Washington DC. After MedStar Health Research Institute Institutional Review Board approval (\#2012-398), we utilized a database of all ectopic pregnancies from January 2009 to December 2012 to identify study candidates. Inclusion criteria were i) diagnosis of an ectopic pregnancy by inappropriate rise in hCG or sonographic findings consistent with ectopic pregnancy and ii) MTX as initial management of ectopic pregnancy. Our exclusion criteria were i) MTX given coincident at the time of surgery and ii) initial surgical management. After patients were identified, we performed a retrospective chart review using the inpatient and outpatient hospital electronic medical records (EMR), Amalga and Centricity. We collected demographic (i.e. race, age, parity, insurance, distance of residence from the hospital) and pertinent presenting clinical data (i.e. past medical history, history of prior ectopic pregnancies, dates, times and locations where hCG levels were drawn, barriers to follow-up (e.g. lack of transportation, work obligations, incarceration), MTX doses and surgical interventions.
Ectopic pregnancies were diagnosed at our institution by i) beta hCG above the discriminatory zone established during the study dates $(1,500-2,000 \mathrm{mIU} / \mathrm{mL})$ without an intrauterine gestational sac (IUGS) visualized on transvaginal ultrasound (TVUS) ii) an adnexal mass on TVUS consistent with an EP without an IUGS and hCG above the discriminatory zone or with inappropriate rise in hCG $(<50 \%)$ over 48 hours or iii) inappropriately rising hCG without an IUGS visualized on TVUS. Prior to giving MTX, all patients underwent a complete history and physical exam, as well as a baseline hCG, complete blood count, basic metabolic profile and liver function tests. Standard of care was the single dose MTX protocol, giving MTX $50 \mathrm{mg} /$ $\mathrm{m} 2 \mathrm{IM}$ on day 1 with recommended follow up hCG levels on day 4 and day 7. Patients were instructed to return to the Emergency Department for the blood draw or were given the option to return to the MWHC Obstetrics and Gynecology outpatient clinic if they were engaged in care there. Per department protocol, patients who were unable to be reached after three or more phone attempts up were sent certified letters.

We calculated protocol adherence by determining the proportion of patients who presented for a repeat hCG on the both days designated by the standard protocol (day 4 and day 7). Alternate protocol adherence was extrapolated by identifying all patients who following up on day 4 for "Day 4" protocol and those patients following up on day 7 for "Day 7" protocol. After examining follow-up trends and reviewing individual cases, we found that many patients followed-up one day later than the protocol designated because of late timing of the MTX dose (e.g. MTX was ordered at $11 \mathrm{pm}$ but the patient did not receive it until 12:30 am) or improper provider counseling (i.e. counting the day of MTX injection as day 0 instead of day 1). Based on these findings, in our analysis of the standard and alternate protocols, we expanded our definition of follow-up on on Day 4 to include patients who had hCG levels drawn on Day 5 , and follow-up on Day 7 to include Day 8.

We used the previously published expected decreases in hCG after single dose MTX to define protocol success, specifically a decrease in hCG from day 4 to day 7 by $15 \%$ for the standard protocol or a decrease in hCG from day 1 to day 4 by $>20 \%$ or decrease in hCG from day 1 to day 7 by $>50 \%$ for the alternate protocols. Conversely, protocol failure was defined as inadequate drop in hCG based on established standard or alternate protocol levels. Loss to follow-up was defined as failure to document a negative urine pregnancy test, or hCG level less than 15 in the medical record within six months after the initial treatment. After surgical intervention, a negative urine pregnancy test or hCG level was not necessary for documentation of completed treatment. Lastly, we evaluated clinical treatment success, defined as documented resolution of pregnancy after a single dose of MTX (i.e. not requiring a second dose of MTX or surgical intervention). 
We performed descriptive statistics using frequency and percentages along with graphs. We tested association between two categorical measures using Chi squared test and Fisher's exact test in case of small cell sizes. For ordinal data, we used Cochran-Armitage to test trends. Analysis is performed using SAS 9.3 by SAS Institute Inc., Cary, NC, USA. To measure and compare the capacity of the protocols to discriminate between protocol success and protocol failure, we analyzed the "Area under the Curve" (AUC) obtained from Receivers Operating Characteristic (ROC) Curve analysis. We compared the AUC of the different protocols using DeLong's test for two ROC curves.

\section{Results}

Of the 149 patients in the EP database, 117 met inclusion criteria. The majority of patients were between 20 and 29 years old $(n=59,50.4 \%)$, African-American $(n=95$, $81.2 \%$ ), without medical co-morbidities ( $n=74,67.9 \%)$, on public health insurance $(n=88,75.2 \%)$ and lived less than 10 miles from the hospital $(n=107,92.2 \%)$, reflecting the inner city demographics of MWHC. Fourteen patients (12.5\%) had a history of a previous ectopic pregnancy; 25 (21.4\%) were established patients in the MWHC Obstetrics and Gynecology clinic (Table 1).

The most commonly reported presenting symptoms were pain $(n=21,18.1 \%)$, bleeding $(n=35,30.2 \%)$, or both $(n=51,44.0 \%)$. Patients were diagnosed with an EP by ultrasound $(n=52,46.9 \%)$ or ultrasound with an inappropriate rise in hCG $(n=57,51.4 \%)$. Two patients (1.8\%) did not have clearly documented diagnostic criteria. The majority of patients had a day 1 hCG of less than $3,000(n=84,71.8 \%)$ and an estimated gestational age of four to eight weeks based on last menstrual period ( $\mathrm{n}=82,70.1 \%$ ). All patients had a day 1 beta hCG prior to receiving MTX.

\section{Protocol Adherence}

Twenty-two (18.8\%) patients were adherent to the standard single dose MTX protocol on day 4 and day 7 . Twenty-four (20.5\%) patients followed up on day 5 and day 8 . Considering these two groups as one, $39.3 \%(n=46)$ were adherent to the standard protocol. We compared patients who followed up days 4 and 7 compared to days 5 and 8 and did not find significant differences in demographic characteristics and clinical outcomes.

Of those patients that did not adhere to the standard MTX follow up protocol on days 4 and 7, 47 patients (40.2\%) presented on day 4 for a second hCG level and 38 patients $(32.5 \%)$ on day 5 . Combining these two days, $71.8 \%$ of patients would have been adherent to the alternate Day 4 protocol having a second hCG level on day 4 (or 5). Similarly, we calculated the proportion of patients that would have been adherent to the Day 7 alternate protocol; 68 patients (58.1\%) patients followed up for a single follow up hCG level on day 7 or 8 . (Figure 1).
Table 1: Demographics

\begin{tabular}{|c|c|c|c|}
\hline Demographics & & $\mathrm{N}$ & Percent \\
\hline \multirow[t]{4}{*}{ Age (years) } & $<20$ & 14 & $12.0 \%$ \\
\hline & $20-29$ & 59 & $50.4 \%$ \\
\hline & $30-39$ & 41 & $35.0 \%$ \\
\hline & $\geq 40$ & 3 & $2.6 \%$ \\
\hline \multirow[t]{4}{*}{ Race } & Black & 95 & $84.1 \%$ \\
\hline & White & 4 & $3.5 \%$ \\
\hline & Hispanic & 13 & $11.5 \%$ \\
\hline & Asian & 1 & $0.9 \%$ \\
\hline \multirow[t]{5}{*}{ Gravity } & G1 & 24 & 20.5 \\
\hline & G2 & 27 & $23.1 \%$ \\
\hline & G3 & 23 & $19.7 \%$ \\
\hline & G4 & 15 & $12.8 \%$ \\
\hline & $\geq \mathrm{G} 5$ & 28 & $23.9 \%$ \\
\hline $\begin{array}{l}\text { History of Ectopic } \\
\text { Pregnancy }\end{array}$ & $\geq 1$ & 14 & $12.5 \%$ \\
\hline \multirow{5}{*}{$\begin{array}{l}\text { Medical } \\
\text { Co-morbidities }\end{array}$} & None & 74 & $67.9 \%$ \\
\hline & HTN & 5 & $4.6 \%$ \\
\hline & Asthma & 8 & $7.3 \%$ \\
\hline & $>1$ problem & 8 & $7.3 \%$ \\
\hline & Unknown & 8 & $6.8 \%$ \\
\hline \multirow[t]{2}{*}{ MWHC Patient } & Yes & 25 & $21.4 \%$ \\
\hline & No & 92 & $78.6 \%$ \\
\hline \multirow[t]{3}{*}{ Insurance } & Public & 88 & $75.2 \%$ \\
\hline & Private & 23 & $19.7 \%$ \\
\hline & Uninsured & 6 & $5.1 \%$ \\
\hline
\end{tabular}

\section{Protocol Success}

Of the $39 \%$ of patients who were adherent to the standard protocol, 69.6\% $(n=32)$ met protocol success with a $\geq 15 \%$ decrease in hCG levels between days 4 (or 5 ) and 7 (or 8 ). Although protocol adherence was higher for patients who would have been adherent to the alternate protocols, protocol success was lower. Of the $71.8 \%$ of patients who would have been adherent to the Day 4 protocol and $58.1 \%$ of patients who would have been adherent to the Day 7 protocol, only $44.1 \%$ and $41.2 \%$, respectively, would have had an appropriate decrease in hCG levels. 
Figure 1: Patient follow-up for their second and third hCG levels after receiving MTX per the standard protocol.

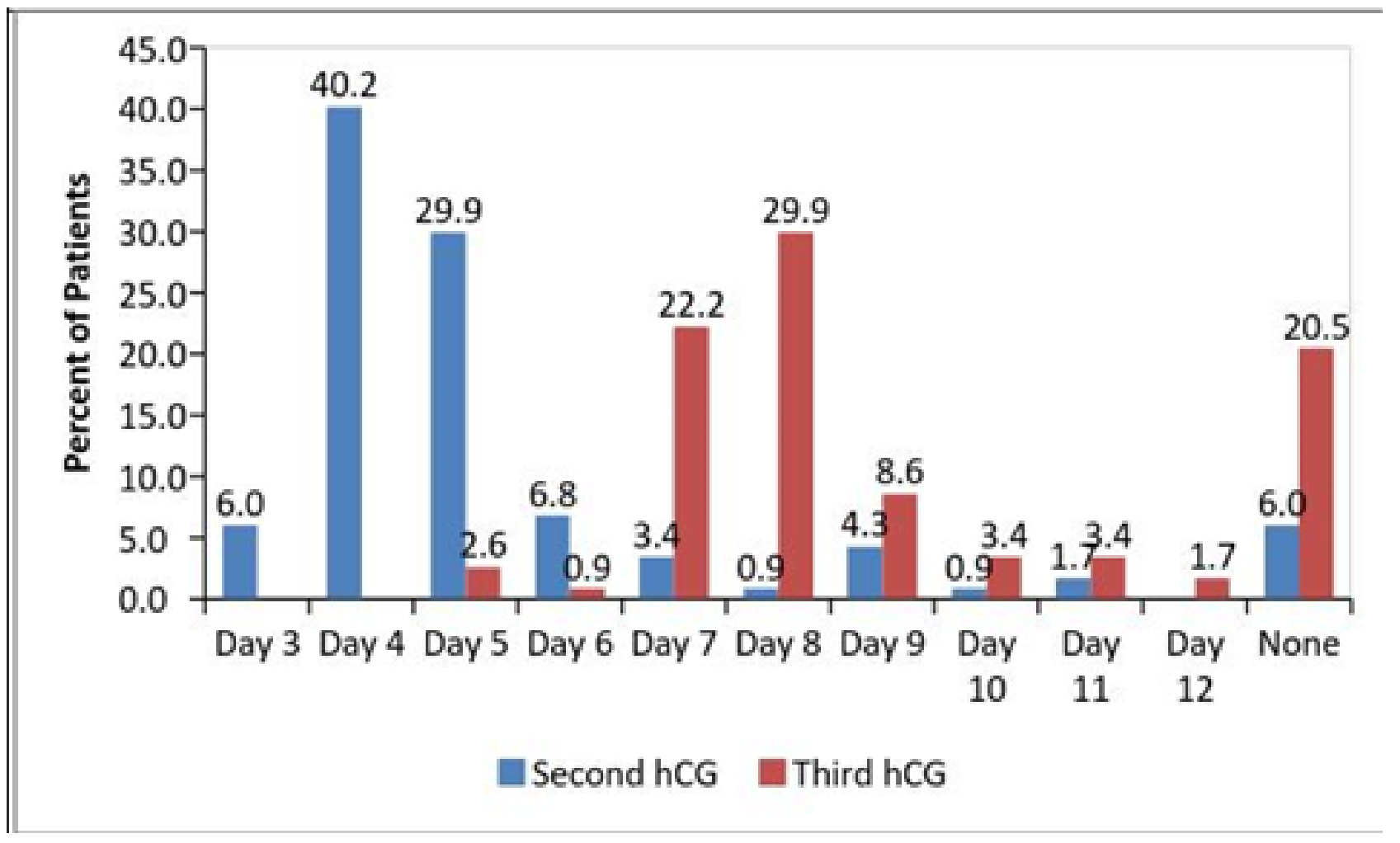

\section{Loss to Follow-up}

A significantly higher proportion of non-adherent patients were eventually lost to follow-up, compared to patients who were adherent to the standard protocol $(31.0 \%$ vs $6.5 \% \mathrm{p}=0.002$ ). Providers most commonly cited work, transportation, incarceration and follow-up at another facility as barriers to follow-up in the medical record. We categorized patients who were lost to follow-up as clinical treatment failures to avoid falsely inflate success rates of protocols.

\section{Clinical Treatment Success}

We did not find a significant difference in receipt of an additional dose of MTX or surgical intervention in patients who were adherent and non-adherent to the standard proto$\operatorname{col}(54.4 \%$ vs. $42.3 \%, \mathrm{p}=0.2)$. We were underpowered to detect such a difference, however, as ad-hoc power analysis revealed that sample sizes of 46 and 71 achieve $26 \%$ power to detect a difference between the group proportions of $12.1 \%$. Standard protocol had an area under the curve (AUC) of 0.558 for discriminating between clinical treatment success and treatment failure, whereas the 'Day 4 protocol' had AUC of 0.629. There was no significant difference between AUCs of these two protocols $(p=0.103)$. Similarly 'Day 7 protocol' had an AUC of 0.604 and it was not significantly different than the AUC of the standard protocol $(\mathrm{p}=0.274)$. The 'Day 7 protocol' and 'Day 4 protocol' AUC's were also not significantly different $(\mathrm{p}=$ 0.6002) (Figure 2).

\section{Clinical Treatment Failures}

In the protocol adherent group, one patient had a ruptured EP on day 3, two patients re-presented for day 4 hCG levels followed by a ruptured EP on day 5 , three patients were considered to be successfully treated and two patients had received a second dose of MTX on day 7 secondary to an inappropriate drop in hCG. In the protocol non-adherent group, three patients did not return after receiving MTX on day 1 until they presented with EP rupture on days 8, 9 and 9. The median day of surgery for ruptured EP was day 19 in the standard protocol group compared to day 9 in the non-adherent group $(\mathrm{p}=0.08)$. A significantly higher proportion of patients in the standard protocol adherent group experienced a ruptured EP $(\mathrm{n}=8,17.4 \%$ vs. $\mathrm{n}=3,4.2 \% ; \mathrm{p}=0.02)$. However, when we excluded patients from the analysis who ruptured before day 5 , and thus did not fully complete the standard protocol, the difference was no longer statistically significant $(p=0.15)$. We did not find a significant difference between the two groups in giving a second dose of MTX or in surgical intervention. In the standard protocol group a second dose of MTX was given 9 times (19.6\%) and in the non-adherent group a second dose of MTX was given 12 times (20.3\%; $\mathrm{p}=0.9)$. In the standard protocol group, 13 patients $(28.3 \%)$ had surgical intervention compared to 9 patients $(15.3 \%)$ in the non-adherent group $(\mathrm{p}=0.1)$. However, ad-hoc power analysis revealed sample sizes of 46 and 59 resulted in a $5 \%$ power to detect a difference between the group proportions of $0.70 \%$ and $36 \%$ power to detect a difference between the group proportions of $13 \%$. 
Figure 2: Patient follow-up for their second and third hCG levels after receiving MTX per the standard protocol.

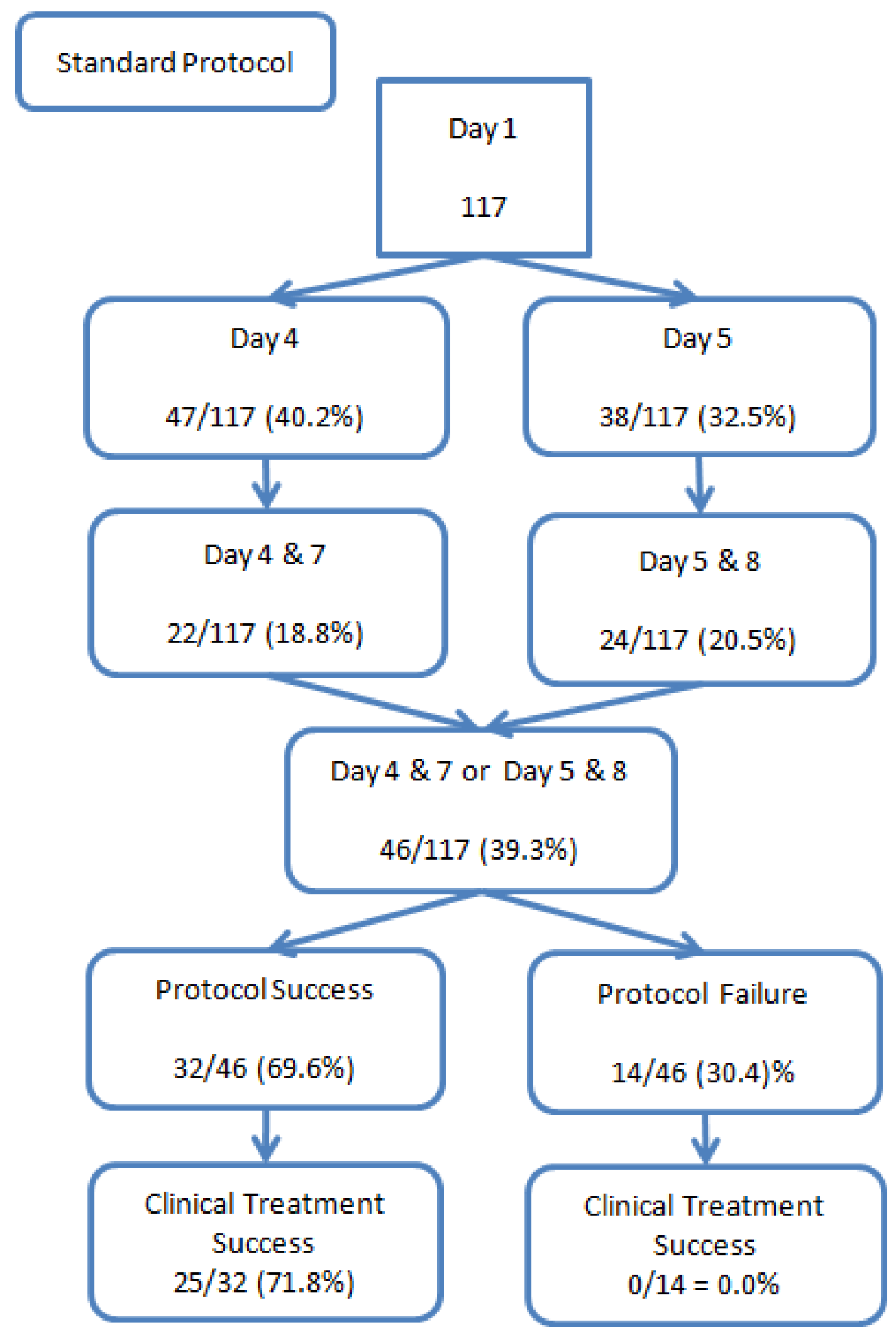




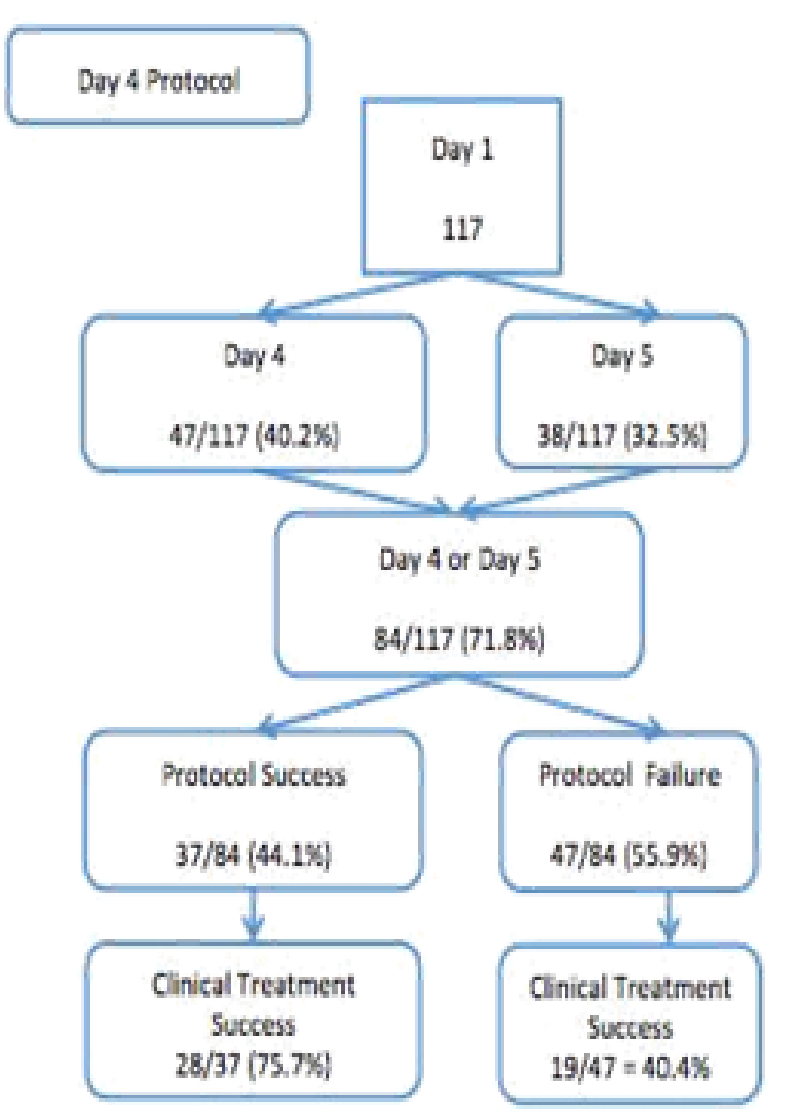

\section{Discussion}

Ectopic pregnancy remains the single most common cause of maternal mortality among black women and among all women in the first trimester in the United States (US). Ectopic-associated mortality is four times higher in blacks and other non-whites than whites, attributed to poor access to care and later detection of EP [4]. A study by Jaspan et al fond that in an inner city US population, less than one in five patients comply with follow-up protocols and $<50 \%$ are followed to confirmed resolution of EP. Moreover, women who were uninsured or on public assistance were almost five times more likely to have treatment failure than those who were insured [2].

This study was motivated by concern for the inconsistent follow up and perceived high rates of loss to followup. Our findings support our hypotheses that the majority of patients were non-adherent to the standard protocol follow up schedule. Adherence to the standard protocol was low at $39.3 \%$, but higher than the previously reported $20 \%$ in an urban population [2]. We may have overestimated the adherence to standard protocol in our population by including patients who followed up on day 5 and day 8 into the standard protocol group. Poor adherence raises the question of whether MTX is an appropriate treatment in this patient population as noncompliance is considered a contraindication to medical management of EP.

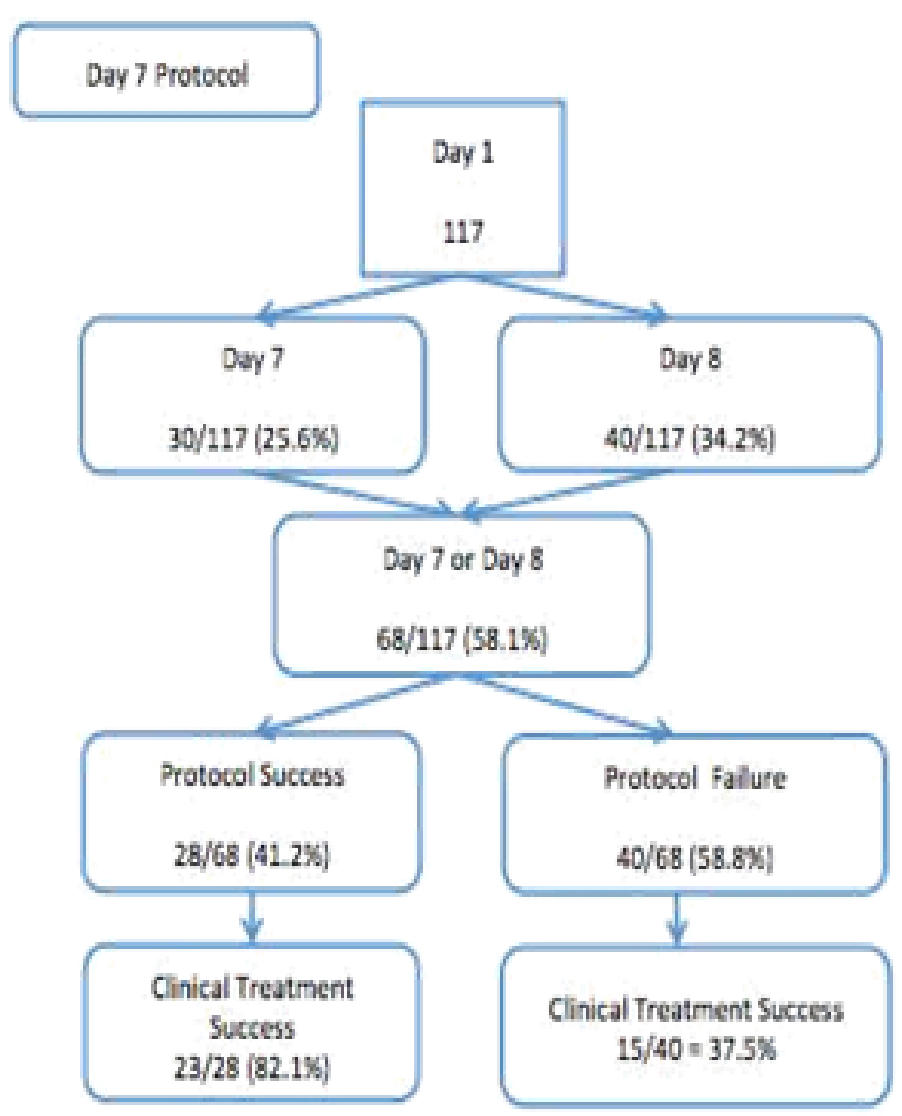

Non-adherence is not only a patient safety concern, it also makes for additional work for the clinicians who contact the patient via phone and letters when they do not present. Although adherence to the standard protocol was low, the overwhelming majority $(73.3 \%)$ of patients did return for at least two follow-up hCG levels. The highly varied, but high proportion of follow-up between days 2 and 12 begs the question of which follow-up protocol best combines the needs for safety, accurate prediction of successful treatment and allows for the greatest patient adherence.

Patients were more likely to present on days 4 or 5 suggesting that the 'Day 4 protocol' would offer the highest patient adherence compared to the 'Day 7 protocol'. When we applied the "Day 4 protocol', the protocol success was significantly lower than for the standard protocol (44.1\% vs. $69.6 \%)$ although the clinical treatment success rates for the two groups were commensurate. The lower protocol success without lower treatment success of the current 'Day 4 Protocol' raises the concern of potentially over-treating patients prematurely with a second dose of MTX or surgery if they did not meet the protocol criteria of $\geq 20 \%$ decrease between days 1 and 4. A reasonable alternative in a clinically stable patient who did not meet protocol success would be to revert back to the standard protocol and require a day 7 hCG level. Almost half of the patients in our study who failed the 'Day 4 protocol' had a subsequent decrease hCG on days 7 or day 8 . About $32 \%$ of the patients who failed the 'Day 4 protocol" necessitated surgical intervention and 27\% necessitated a second dose of MTX. 
The 'Day 7 protocol' may also offer a reasonable alternative to the standard protocol with similar protocol and treatment success rates as the 'Day 4 protocol.' The major limitation of Day 7 protocol is the need for a second dose of MTX or surgery on those that are stable but fail to decrease their hCG levels per the 'Day 7' protocol since data is not available on continued observation of these patients.

Our proportion of protocol success was lower than what is routinely quoted in the medical literature $(69.6 \%$ vs. 88.1\%) [5]. Our cohort did not demonstrate known risk factors for failure such as initial hCG level $>5000 \mathrm{mUI} / \mathrm{mL}$, sac size $>4 \mathrm{~cm}$, fetal cardiac activity, rapidly rising hCG immediately preceding treatment with MTX and continued rapid rise in hCG after initial MTX dose [10]. Mean day 1 hCG level for all treatment failures was $2665 \mathrm{mIU} / \mathrm{mL}$ (SD \pm 1995$)$, which was slightly higher than the $2059 \mathrm{mIU} / \mathrm{mL}(\mathrm{SD} \pm 2030)$ for those successfully treated $(p=0.08)$. Further research is necessary to understand the disparity in protocol success in our patient population. We found no statistical significant difference in rates of ruptured EPs between the standard protocol group versus non-adherent group or those adherent to the 'Day 4 protocol' versus the 'Day 7 protocol'. However, we were not powered to see a difference between the groups since the given the low risk of a rupture (between $7 \%$ and $14 \%$ ) $[9,11]$.

This study is limited by its retrospective and descriptive design as well as small sample size and thus limited power to detect some differences. The potential sample size for analysis is further reduced with the high loss to follow up rate which is characteristic of poor urban communities [2].

\section{Conclusions}

We found that the current standard follow up for single dose MTX after treatment of an EP does not meet the needs of our unique patient population given the poor adherence rates. In our small sample size, morbidity from patient driven follow up patterns was not increased. Alternate follow up options, such as the 'Day 4' and 'Day 7' protocols appear safe and effective and should be further explored as an adjunct to the current standard protocol especially in this patient population. Alternative follow-up protocols may offer patients greater flexibility without compromising patient care [2,6-8].

\section{References}

1) American College of Obstetricians and Gynecologists. (2008) Practice Bulletin 94: Medical Management of Ectopic Pregnancy. American College of Obstetricians and Gynecologists.

2) Jaspan D, Giraldo-Isaza M, Dandolu V, Cohen AW (2010) Compliance with Methotrexate therapy for presumed ectopic pregnancy in an inner-city population. Fertil Steril; 94:1122-11224.

3) Agostini A, Blanc K, Rhonda I, Romain F, et al. (2007). Prognostic value of hCG changes after methotrexate injection for ectopic pregnancy. Fertil Steril; 88: 504-506.

4) Centers for Disease Control and Prevention (CDC) (1995) Ectopic pregnancy--United States, 1990-1992. MMWR Morb Mortal Wkly Rep. 44: 46-48.

5) Alleyassin A, Khademi A, Aghahosseini M, Safdarian L, Badenoosh B, et al. (2006) Comparison of success rates in the medical management of ectopic pregnancy with single dose and multiple dose administration of methotrexate: A prospective, randomized trial. Fertil Steril 85: 1661-1666.

6) Butts SF, Gibson E, Sammel MD, Shaunik A, Rudick B (2010) Race, socioeconomic status and response to methotrexate treatment of ectopic pregnancy in an urban population. Fertil Steril 94: 2789-2792.

7) Thurman AR, Cornelius M, Korte JE, Fylstra DL (2010) An alternative monitoring protocol for single-dose methotrexate therapy in ectopic pregnancy. AJOG 202: 139.e1-6.

8) Menon S, Colins J, Barnhart KT (2007) Establishing a human chorionic gonadotropin cutoff to guide Methotrexate treatment of ectopic pregnancy: a systematic review. Fertil Steril 87: 481-484.

9) Lentz G, Lobo R (2012) Comprehensive gynecology. St. Louis, Mo: Mosby.

10) Practice Committee of American Society for Reproductive Medicine (2008) Medical treatment of ectopic pregnancy. Fertil Steril 90: 206-212.

11) Dudley PS, Heard MJ, Sangi-Haghpeykar H, Carson SA, et al. (2004) Characterizing ectopic pregnancies that rupture despite treatment with methotrexate. Fertil Steril; 82: 1374-1378.

Submit your manuscript to a JScholar journal and benefit from:

ฯ Convenient online submission

ब Rigorous peer review

ब Immediate publication on acceptance

๑ Open access: articles freely available online

- High visibility within the field

- Better discount for your subsequent articles

Submit your manuscript at

http://www.jscholaronline.org/submit-manuscript.php 